\title{
MENTHOL-CARBOXYLIC ACID DEEP EUTECTIC SOLVENT FOR EXTRACTION OF CAROTENOIDS FROM PALM ESTER
}

\author{
R. Manurung, A.G.A. Siregar ${ }^{\varpi}$ and R. Hasibuan \\ Department of Chemical Engineering, Faculty of Engineering, Universitas Sumatera Utara, \\ Medan, Indonesia, 20155. \\ Corresponding Author: alwigery10@gmail.com
}

\begin{abstract}
In this study, the carotenoids in palm biodiesel will be extracted using methanol and deep eutectic solvent (DES) as a co-solvent using the solvent extraction process. The DES used is menthol-based as a hydrogen bond donor and acetic acid and lauric acid hydrogen bond acceptor (HBA) and, which is correlated with a molar ratio of 1: 1 and 2: 1. To see the functional groups formed, FTIR characterized the DES generated while seeing the structure of the components formed in DES using $\mathrm{H}^{1} \mathrm{NMR}$. To see the $\beta$-carotene portion obtained, complete carotenoids were characterized by the UV-Visible Spectrophotometer, HPLC. The optimum condition for complete carotenoid extraction using DES menthol is $366.3 \mathrm{ppm}$ : lauric acid with an ester: DES ratio of 1: 0.5 . Whereas the content of $\beta$ carotene derived from DES menthol is $14.7 \mathrm{ppm}$ lauric acid. These findings show that the total extraction yield of carotenoids and $\beta$-carotene in palm methyl esters can be increased by DES as a co-solvent.
\end{abstract}

Keywords: Carboxylic Acid, Deep Eutectic Solvent, Methyl Ester

RASĀYAN J. Chem., Vol. 14, No.3, 2021

\section{INTRODUCTION}

Biodiesel is a renewable, biodegradable, and non-toxic fuel generated from oil or fat through an alcohol transesterification reaction. Palm oil crude biodiesel includes by-products called minor ingredients, consisting of FFA, monoglycerides (MG), diglycerides (DG), triglycerides (TG), and antioxidants such as carotenoids $(\alpha-, \beta$-carotene) and vitamin $\mathrm{E}$ (tocopherol and tocotrienol). Minor components of crude palm oil contain 500-700 ppm carotenoids, over 80 percent in the form of $\alpha$-, $\beta$-,-carotene with $\beta$-carotene as the main component ${ }^{1}$. $\beta$-carotene as a minor component of crude palm oil is a precursor to vitamin $\mathrm{A}$ and serves as a pro-vitamin $\mathrm{A}^{2}$. $\beta$-carotene has $100 \%$ vitamin A activity while carotene has $50-54 \%$ vitamin A activity. Palm oil carotenoid compounds have a provitamin A activity 10 times higher than carrots and 300 times higher than tomatoes ${ }^{3}$. $\beta$-carotene has positive biological effects that are beneficial to the body, including preventing the onset of cancer, coronary heart disease, preventing premature aging $^{4}$, and can also serve as an antioxidant that kills free radicals that further decreases the risk of developing degenerative diseases ${ }^{5}$.DES is a new generation solvent that is usually formed from a mixture of solid salt and various hydrogen bond donors with different measurement ${ }^{12}$. DES has the advantage of being non-volatile and non-flammable compared to conventional organic solvents, making storage simpler. DES has a melting point lower than each of its components ${ }^{6}$. By understanding the advantages of carotenoid compounds and the high content of carotenoid compounds in crude palm oil, the process of extracting carotenoids from crude palm oil has received considerable attention from researchers. Various methods have been developed for the processing of carotenoid compounds from crude palm oil. Some of the methods used to obtain carotenoids from crude palm oil are lathering, solvent extraction, adsorption, urea, molecular distillation, iodine, membranes, and molecular distillation ${ }^{7}$. A study on the isolation of carotenoids from crude palm oil was carried out using a solvent extraction method using an alcohol solvent and a deep eutectic solvent-based on menthol as a co-solvent. Furthermore, a study was conducted to release the solvent impurities in carotene by washing and drying methods.

\section{Transesterification of CPO}

\section{EXPERIMENTAL}

$\mathrm{KOH}$ with a weight of $1 \%$ of 50 grams of palm oil reacts with ethanol to form a homogeneous solution of potassium methoxy. After that, potassium methoxy was applied to CPO: ethanol at a ratio of 1:9 in a Rasayan J. Chem., 14(3), 1869-1874(2021)

http://doi.org/10.31788/RJC.2021.1436293

This work is licensed under a CC BY 4.0 license. 
three-cock flask at a speed of $300 \mathrm{rpm}$ at a temperature of $60{ }^{\circ} \mathrm{C}$ for 1 hour. The methyl ester compound is isolated by glycerol into a separate funnel for 8 hours. The upper layer is methyl ester, while the lower layer is glycerol. The methyl ester was washed with a volume of $300 \mathrm{ml}$ of water.

\section{Preparation of DES}

Menthol with acetic acid and lauric acid with a molar ratio of $1: 1$ and $2: 1$ reacts at a temperature of $50{ }^{\circ} \mathrm{C}$ for 15 minutes with a rotation of $300 \mathrm{rpm}$ to form a homogeneous and transparent solution ${ }^{8}$. In this analysis, the hydrogen bond acceptor originated from Menthol, while the hydrogen bond donor (HBD) was derived from carboxylic acid compounds, namely acetic acid and lauric acid with different molar ratios with code DES 1 and DES 2.

\section{Extraction of Carotenoids From Methyl Esters}

10 grams of methyl ester obtained from the transesterification process are mixed with $40 \mathrm{ml}$ of ethanol and DES with a ratio of 1: 0.5 (W/W) (Ester: DES). Then the mixture is stirred with a stirrer for 3 hours at a speed of $200 \mathrm{rpm}$ at room temperature. Then the product formed is separated which will form two layers where the top layer is ethanol and the remaining DES and vitamin E components while the bottom layer is carotene, DES, and the remaining ethanol solvent. The bottom layer formed was washed with ethanol: water with a ratio of $1: 2(\mathrm{v} / \mathrm{v})$ for 10 minutes to remove the remaining DES residue in the carotene product ${ }^{9}$. Furthermore, the drying process is carried out at a temperature of $50-60 \mathrm{C}$ for 3 hours to remove the water content and the remaining DES.

\section{DES and Methyl Ester and Carotene Analysis Characterization}

The Formed DES clear liquid was then characterized by a Shimadzu FTIR with a wavelength of 400-4000 $\mathrm{cm}^{-1}$ to see the functional groups of the compounds formed in the DES mixture. $\mathrm{H}^{1} \mathrm{NMR}$ analysis is used to see the structure and composition of the compounds in the DES solution. Analysis of ester content as raw material using GC-MS and carotenoid content using a UV-Visible spectrophotometer. The total content of carotenoids extracted using UV-Visible Spectrophotometer analysis. Meanwhile, $\beta$-carotene compounds use HPLC.

\section{Preparation of DES}

\section{RESULTS AND DISCUSSION}

DES was prepared by mixing salt and hydrogen bond donor (HBD) compounds that are both linked by hydrogen bonds ${ }^{6}$. The reason it is called DES is that when two components are combined in the right ratio, the eutectic point of the mixture is obtained ${ }^{9}$. The mechanism of the bond between the Hydrogen Bond Acceptor and the Hydrogen Bond Donor (R-OH) is shown in (Fig.-1). The bond between HBA and HBD occurs by hydrogen bonding between salt halide anions and hydrogen donor groups.

\section{FTIR Analysis}

The carbonyl group study of the material is carried out by comparing the absorption bands formed in the infrared spectrum using a correlation table by using a range of comparable compounds. FTIR study of DES compounds was carried out to classify variations in classes of DES compounds. From a functional group analysis using FTIR, the results of the spectrum in graphical form are shown in (Fig.-2).
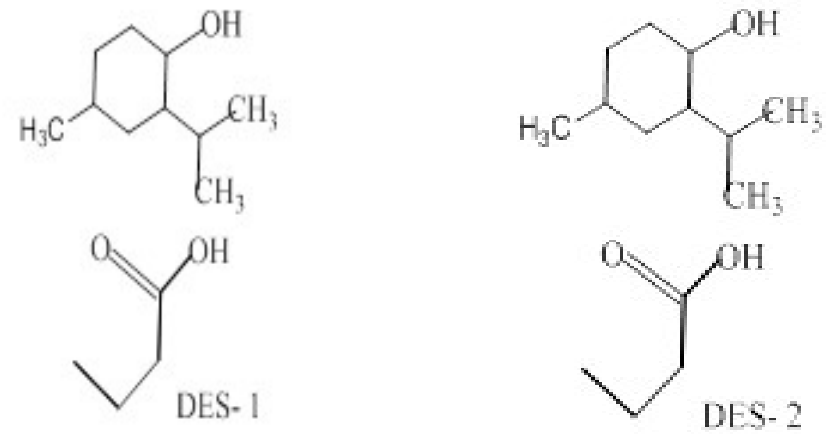

Fig.-1: DES 1 and DES 2 

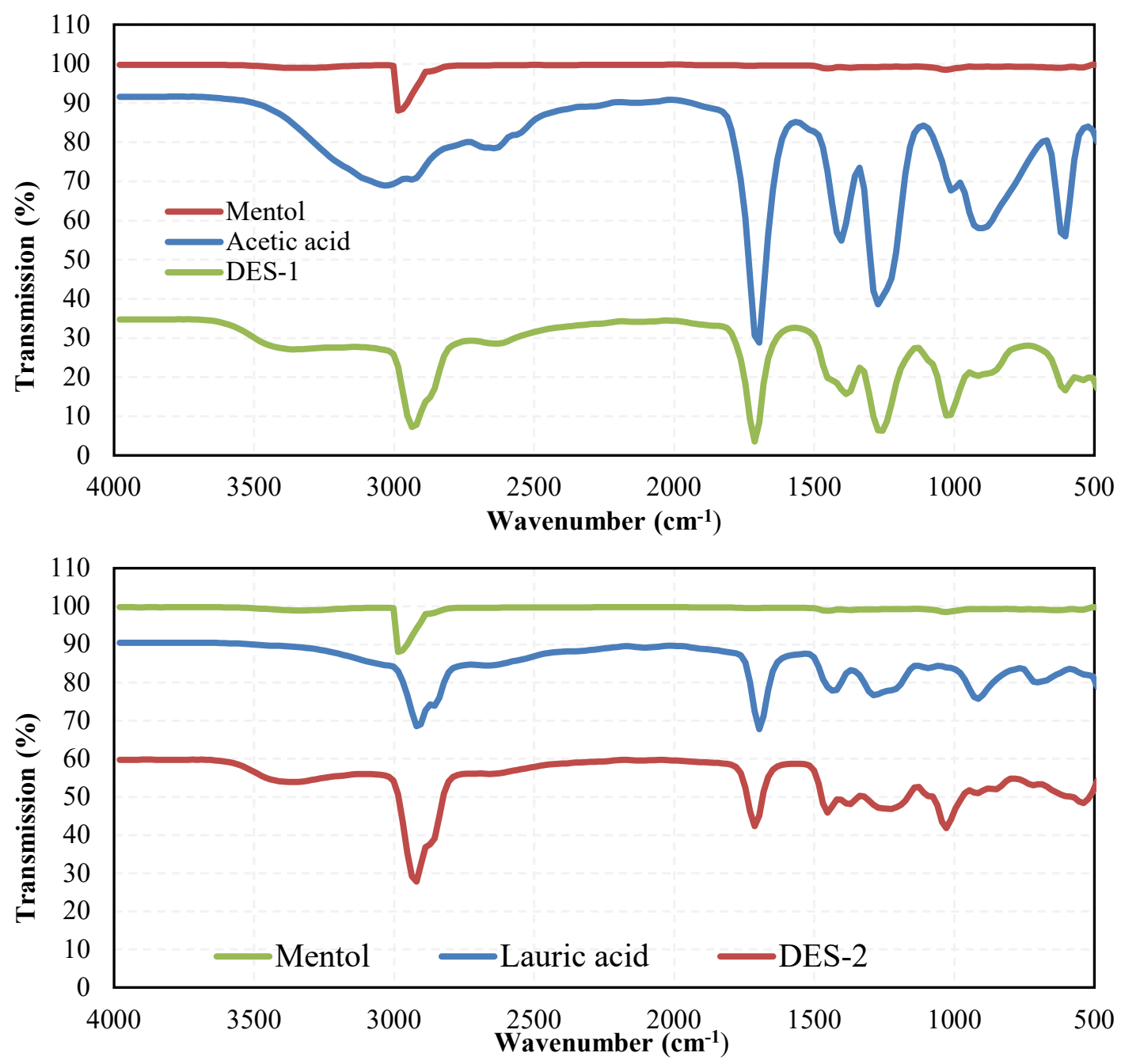

Fig.- 2: FTIR DES 1 and 2 Spectra

Both hydrogen bond donors used in this eutectic mixture have a carboxylic acid group in their structure, which represents a representative band (ketone or carbonyl group) around the $1700 \mathrm{~cm}^{-1}$ wavenumber of the spectrum. FTIR spectrum of the hydrogen bond acceptor, menthol, only one representative band corresponds to the hydroxyl group at approximately $3320 \mathrm{~cm}^{-1}$. Besides, FTIR measurements are also used to validate the formation of new compounds through the formation of hydrogen bonds between menthol and hydrogen bond donors. Eutectic mixture FTIR spectra data showed that intermolecular hydrogen bonding interactions between hydrogen bonding menthol donors were mainly in acetic acid and lauric acid. Fig.-2 shows that for menthol: eutectic mixed acetic acid, the HBD carbonyl band is initially at lower wavenumber $\left(\sim 1692 \mathrm{~cm}^{-1}\right)$ and expands and moves to the maximum number (1711.11 and $1711.21 \mathrm{~cm}^{-1}$ ) in the eutectic mixture ${ }^{10}$. This is a clear sign that hydrogen bonds are forming, which means that a new compound is forming. The physical state of the compounds may further confirm this proof, e.g. the eutectic mixture of menthol and lauric acid: initially, the physical state of the two compounds is solid and a transparent liquid is obtained after the formation of DES.

\section{$\mathbf{H}^{1}$ NMR Analysis}

The NMR is used to determine the structure of new natural and synthetic components, the purity of the components, and the direction of chemical reactions, and the relationship between components in a solution that can undergo chemical reactions. Proton spectrophotometry or $\mathrm{H}^{1} \mathrm{NMR}$ provides structural information about the hydrogen atoms in an organic molecule. Not all $1 \mathrm{H}$ nuclei reverse their spin exactly 
equal to frequency because they may differ in their chemical or even electronic environments. This condition causes a chemical shift. The results of the $\mathrm{H}^{1} \mathrm{NMR}$ DES 1 and DES 2 spectrophotometry in this study are presented (Fig.-3).
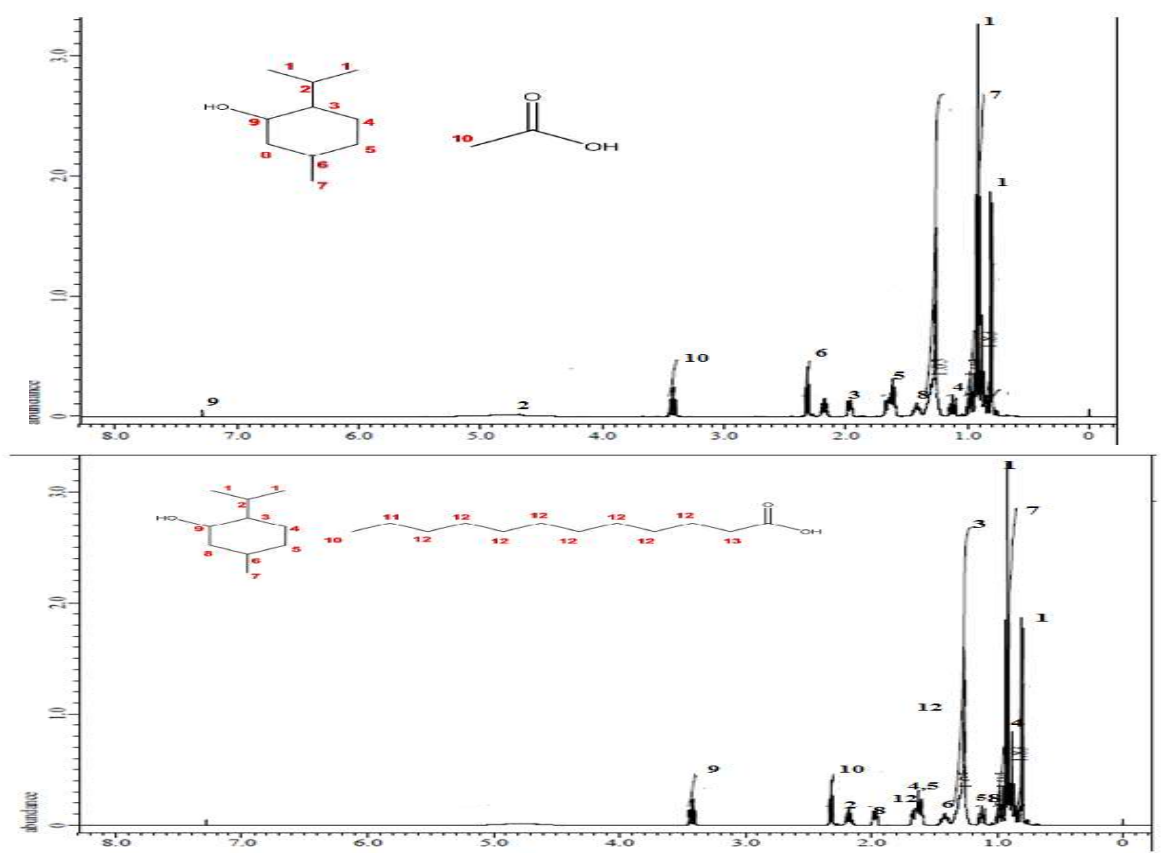

Fig.-3: H1NMR DES 1 and 2 Spectra

In the $\mathrm{H}^{1} \mathrm{NMR}$ spectrum above, DES solutions have formed with an enlarged spectrum that accumulates and spreads in the chemical environment of menthol compounds, acetic acid, and lauric acid. This proves that hydrogen bonding has occurred between HBA and HBD.

\section{Analysis of Raw Material for Methyl Ester from Crude Palm Oil (CPO)}

In this research, methyl ester comes from crude palm oil (CPO) through the transesterification process. The catalyst used was $\mathrm{KOH}$ while the solvent used was ethanol. The resulting methyl ester product was characterized using gas chromatography to see the methyl ester content obtained, while the amount of carotene was characterized by a UV-Visible Spectrophotometer with a wavelength method of $470 \mathrm{~nm}$. The results of esters and carotenoids are presented in Table- 1 .

\section{Effect of Methyl Ester: DES Ratio on Carotene Concentration}

Most carotenoids are hydrophobic, lipophilic compounds that are insoluble in water but are soluble in fat solvents. In animals and plants, carotenoids are crystalline or amorphous solid and fat-soluble. The poor solubility of DES in water shows that DES in this study is a non-polar molecule, or hydrophobic. In contrast, methyl esters are non-polar organic substances. This information is important for making a biphasic system in liquid-liquid extraction ${ }^{11}$. In this study, DES acts as a co-solvent by using the main solvent of ethanol, which is semi-polar. Using ethanol in this study is because it is an organic compound, so it is non-toxic and does not pollute the environment compared to the use of a polar compound, namely hexane. The results of the carotene concentration obtained are shown in (Fig.-4).

Table-1: Characteristics of Methyl Ester

\begin{tabular}{c|c|c}
\hline No & Parameter & Methyl Ester \\
\hline 1 & Triglyceride (\%) & 37.2376 \\
\hline 2 & Diglyceride(\%) & 3.6250 \\
\hline 3 & Monoglyceride (\%) & - \\
\hline 4 & Ester (\%) & 56.96446 \\
\hline 5 & Carotene (ppm) & 203.3 \\
\hline \multicolumn{3}{|c}{1872}
\end{tabular}




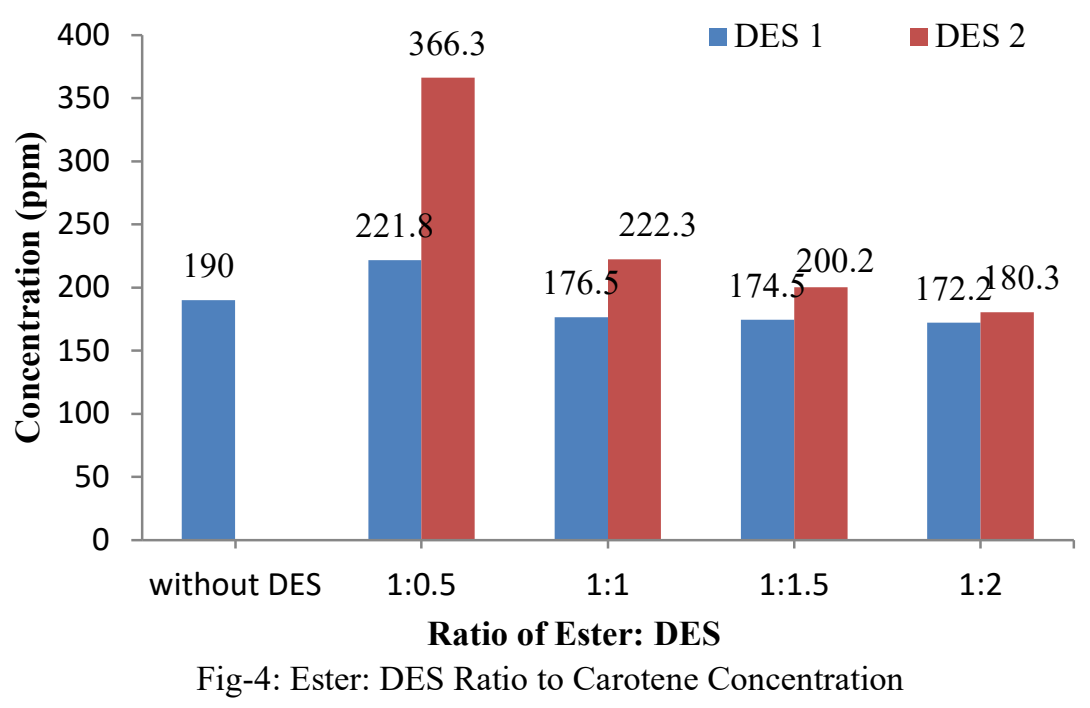

Figure-4 shows the effect of the ester: the DES ratio on the carotene concentration obtained in the ester by extraction showing that the maximum concentration was $366.3 \mathrm{ppm}$ in DES 2 compared to $316.2 \mathrm{ppm}$ in DES 1. This is because the number of carboxylic bonds is more in DES. 2 which is composed of HBD lauric acid $\left(\mathrm{C}_{12} \mathrm{H}_{24} \mathrm{O}_{2}\right)$ compared to DES 1 which is composed of HBD acetic acid $\left(\mathrm{C}_{2} \mathrm{H}_{4} \mathrm{O}_{2}\right)$ which has a lower carboxylic bond. Besides, the extraction treatment without DES (ethanol only) was carried out by producing a carotene concentration of $190 \mathrm{ppm}$. These results indicate that the addition of DES as a cosolvent can increase the concentration of carotene products in the ester. DES polarity is an important factor in deciding the separation efficiency that is determined by the interaction between the solute carotene and DES ${ }^{11}$ Here the polarity of the DES is hydrophobic and becomes the driving force for the transfer of solutes (carotene).

\section{Effect without DES and Addition of DES on $\beta$-Carotene Concentration}

As a minor component of crude palm oil, $\beta$-carotene is a precursor to vitamin A and serves as a provitamin A. $\beta$-carotene has positive biological effects that are beneficial to the body, such as overcoming xerophthalmia blindness, avoiding cancer, coronary heart disease, preventing an aging phase that is too early to improve body immunity and may also serve as an antioxidant ${ }^{13}$. The results of the effect without DES and the addition of DES on $\beta$-carotene concentration show in (Fig.-5) and show that the highest $\beta$ carotene concentration is at $14.7 \mathrm{ppm}$ in DES 2. This shows that the use of DES 2 (menthol: lauric acid) can increase the concentration of $\beta$-carotene compared to DES and the use of DES $1^{12}$.

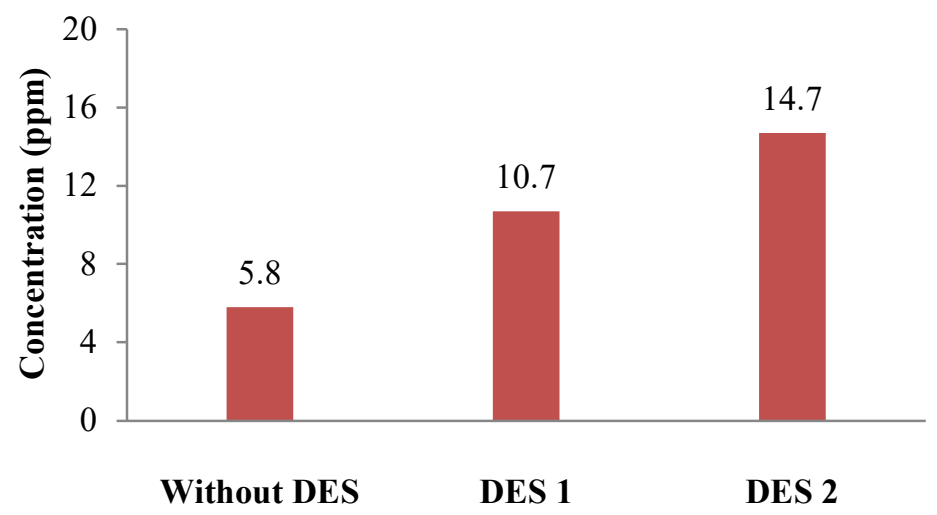

Fig.-5. Effect of Temperature of Calcination on Methyl ester Yield 
RASĀYAN J. Chem.

Vol. 14 | No. 3 |1869-1874| July - September | 2021

\section{CONCLUSION}

The optimum carotene concentration obtained in this study was $366.3 \mathrm{ppm}$ or equivalent to a concentration of 1.80 times using DES 2, It has better polarity than DES 1. This also applies to the concentration of $\beta$-carotene leveraging $14.7 \mathrm{ppm}$. This shows that DES as a co-solvent can increase the concentration of total carotenoid leaching as an environmentally friendly and non-toxic solvent.

\section{ACKNOWLEDGEMENT}

The authors gratefully acknowledge the Ministry of Research and Technology/ National Agency for research and Innovation of Indonesia.

\section{REFERENCES}

1. S.H. Goh, Y.M. Choo, A.S.H. Ong, Journal of the American Oil Chemists' Society, 62, 237(1985), https://doi.org/10.1007/BF02541384

2. T. Grune, G. Lietz, A. Palou, A.C. Ross, W. Sthal, G.Tang, D.Thurnham, S. Yin, H.K Biesalski, The Journal of Nutrition, 140(12), 2268S(2010), https://doi.org/10.3945/jn.109.119024

3. B. Nagendran, Y.M. Choo, U.R. Unnithan, Food and Nutrition Bulletin, 21(2),189(2000), https://doi.org/10.1177/156482650002100213

4. K. Umegaki, S. Ikegami, The American Journal of Clinical Nutrition, 59(2),409(1994), https://doi.org/10.1093/ajen/59.2.409

5. V. Lobo, A. Patil, A. Phatak, N. Chandra, Pharmacognosy Review, 4(8), 118(2010), https://doi.org/10.4103/0973-7847.70902

6. H. Zhao, Z. Cheng, D. Tanisha, Crittle, Journal of Molecular Catalysis B Enzymatic, 85-86, 243(2013), https://doi.org/10.1016/j.molcatb.2012.09.003

7. Y.M. Choo, Food and Nutrition Bulletin, 22, 1(1989), https://doi.org/10.1177/156482659401500212

8. A.H. Nu'man, N. Mei Han, C. Mohd Ali, H. Natesan, Journal of the American Oil Chemists' Society, 92, 11(2015), https://doi.org/10.1007/s11746-015-2720-6

9. H. Zhao, S. Xia, P. Ma, Journal of Chemical Technology \& Biotechnology, 80, 1089(2005), https://doi.org/10.1002/jctb.1333

10. R. Bernardo Dias, F. Catarina, C. I. Lucas, Z. C. Maria Alice, M. M. Isabel, ACS Sustainable Chemical \& Engineering, 3, 10, (2015), https://doi.org/10.1021/acssuschemeng.5b00532

11. H. Schwartz, V. Ollilainen, V. Piironen, A-M. Lampi, Journal of Food Composition and Analysis, 21, 2 152(2008), https://doi.org/10.1016/j.jfca.2007.07.012

12. R. Manurung, Alexander, Rasayan Journal of Chemistry, 11(4),1519(2020), https://doi.org/10.31788/RJC.2018.1143079

13. R. Ziegler, Journal of Nutrition, 119, 1,116(1989), https://doi.org/10.1093/jn/119.1.116

[RJC-6293/2020] 